\title{
Big Data in Finance
}

\author{
Andrey L. Bulgakov, \\ department of finance, Faculty of Economics, HSE: \\ 119049, Moscow, Shabolovka, 26, building 4
}

Keywords: Big data, financial sector, education, management, economic decision-making

JEL: O31, O39

\begin{abstract}
The question of Big Data technologies, not only in the financial sector, but in general, is a logical pattern of technical and scientific progress of the last decades. The change of paradigms has led to the fact that today's managers and economists have to work not only with large volumes of information, but also with new types of data. Processing the new format files allows managers to make more accurate and effective financial decisions. It is necessary to say, why these decisions are important for business. They make it possible to achieve the goals that modern organizations set for themselves: increasing the value of the enterprise, increasing investment attractiveness, improving the quality of forecasting. This situation in a well-func-
\end{abstract}

tioning world system has led to the emergence and development of new trends in education, in particular, in the field of education of financial professionals. Focusing on Russian achievements and Western researches of fundamental and applied disciplines, it should be noted that the modern financier is increasingly integrated into the Big Data technology environment. He needs knowledge of the construction of wording of requests to manage competently. For this, the skills of working with search programs, the use of search operators, work with search robots are important. At the theoretical level the future financier must study strategic, statistical, mathematical, systemic, stochastic, probabilistic and other types of analysis.

\section{Big Data в финансах}

\section{Булгаков Андрей Леонидович,}

кандидат экономических наук, департамент финансов, экономический факультет НИУ ВШЭ: 119049, Москва, ул. Шаболовка, д. 26, корп. 4

Ключевые слова: большие данные, финансовый сектор, образование, управление, принятие экономических решений

JEL: O31, O39

\section{Аннотация}

Вопрос применения Big Data не только в финансовом секторе, но и в целом является логичной закономерностью технического и научного прогресса последних десятилетий. Смена парадигм привела к тому, что сегодняшним менеджерам и экономистам предстоит работать не только с большими объемами информации, но и с новыми типами данных. Обработка файлов нового формата позволит руководителям принимать более точные финансовые решения. Необходимо сказать, почему для бизнеса важны эти решения. Они позволят достичь целей, которые современные организации ставят перед собой: повышение стоимости предприятия, увеличение инвестиционной привлекательности, совершенствование качества прогнозирования. Эта ситуация в хорошо работающей мировой системе привела к появлению и развитию новых трендов в образовании, в частности в сфере обучения финансовых специалистов. Ориентируясь на российские достижения и западные исследования фундаментальных и прикладных дисциплин, следует отметить, что современный финансист все больше интегрируется в среду технологий Big Data, для грамотного управления которыми ему необходимы знания построения формулировки запросов. Для этого важны навыки работы с поисковыми программами, применения поисковых операторов, работы с поисковыми роботами. На теоретическом уровне будущий финансист должен изучать стратегический, статистический, математический, системный, стохастический, вероятностный и другие виды анализа. 
Основной вопрос применения технологий Big Data в финансовом секторе заключается в том, насколько работа с ними позволит увеличить производительность, избежать рисков, прогнозировать и расширять возможности компаний. На самом деле грамотное использование Big Data способно изменить и уже меняет финансовую систему в целом. Необходимо признать, что большие данные всецело стали частью жизни мирового общества. В этих условиях задачей университетов является обучение специалистов навыкам работы с массивами данных и приоритизации данных, умению грамотно формировать запрос и возможностям применения технологий. Главным вопросом, помимо понимания анализа и обработки данных, остается понимание, для чего финансист работает с этими данными, какого результата он может достичь. Современному специалисту в области финансов необходимо понимать суть работы технологий, когда речь идет о Big Data. Для этого необходимо обратиться к истории развития и применения этих технологий. В 1960-х гг. впервые появились суперкомпьютеры (супер-ЭВМ) - вычислительные машины, которые обладают самой большой мощностью в сравнении с другими компьютерами.

Как правило, суперкомпьютер состоит из нескольких серверных компьютеров, соединенных высокоскоростной магистралью. Тогда же впервые были запущены решения векторной обработки, далее - матричные решения, векторно-конвейерные, расширялись возможности оперативной памяти и т.д. Весь процесс модернизации был направлен на решение вопроса распределения, кодирования и хранения информации. Машины стали использоваться в управлении технологическими процессами в производстве, значительно расширилась сфера применения супер-ЭВМ, в большей степени за счет развития программного обеспечения. Тогда же эти мощные вычислительные машины впервые стали использоваться для решения экономических задач, управления процессами производства, передачи информации.

Вычислительные машины в этот период применялись главным образом для обработки больших объемов информации и решения задач, которые связаны с рутинными процессами на производстве. Эти ЭВМ работали по принципу пакетной обработки данных, который, по сути, являлся автоматизацией ручных методов обработки. Впервые широкое распространение получила профессия программиста. Сложилось понимание, что работа с ЭВМ требует аккумуляции усилий специалистов узкого профиля: экономистов, математиков, программистов. Экономист формулировал задачу, математик писал алгоритм, и программист реализовывал этот алгоритм.

Каждые 10 лет происходило стремительное развитие суперкомпьютеров. Появились малые интегральные схемы (МИС), значительно улучшались характеристики ЭВМ, такие как объем оперативной памяти, быстродействие, надежность, при этом уменьшались требуемая мощность, масса и занимаемая площадь. Начали производить мини-ЭВМ и персональные ЭВМ (ПЭВМ), произошло резкое снижение цен на аппаратное обеспечение. Этот период также характеризуется началом применения новых методов программирования, созданием новых программных продуктов и методов планирования. Происходит бурное развитие ПЭВМ реального времени. На ПЭВМ в производстве возлагаются задачи управления подсистемами и обработки центральных данных об объекте. ЭВМ внедряются на заводах, в банках и т.д. ПЭВМ все чаще используются в коммерческой обработке данных для решения коммерческих задач. Надо сказать, что это позволило специалисту, использующему ПЭВМ, совместить работу экономиста, математика и программиста. Однако очевидно, что это резко снизило эффективность и качество работы, поскольку реализация данного процесса требовала наличия определенных профессиональных знаний.

С 1991 г. появился широкополосный Интернет. Его основное преимущество заключалось в том, что он позволял увеличивать скорость передачи данных, а также обеспечивать передачу одновременно нескольких типов данных в один момент времени. Не стоит забывать и о том, что он обладал значительно большей мощностью. Обеспечивал соединение точек на более дальних расстояниях, делал возможным осуществление телеметрических услуг (удаленные измерения и сбор данных с удаленных датчиков, промышленного оборудования в самых разных индустриях). Очевидно, что в этих условиях количество получаемой и создаваемой информации резко возросло. На производстве или в процессе управления также использовались не один, а несколько ЭВМ. Это способствовало дальнейшему усложнению хранения данных и способов доступа к ним. Появились не только локальные сети, но и новые носители информации. Начали подниматься вопросы о надежности хранения данных и активном использовании одновременно больших объемов сохраненной информации. Таким образом, сформировались два основных подхода к хранению данных: на внешнем носителе и в системе. Можно разделить использование организацией этих данных на три этапа:

1. устройства и системы с произвольным доступом для активно используемых данных - для них характерны небольшое время доступа и наиболее высокие значения частоты обращения и удельной стоимости хранения;

2. устройства и системы с произвольным доступом для периодически используемых данных - они занимают промежуточное положение по скорости доступа, емкости и частоте обращения;

3. устройства и системы с последовательным доступом для долговременного хранения данных обращение к ним осуществляется достаточно редко, они медленнее систем хранения первого и второго уровней, обладают наибольшей емкостью и наименьшей удельной стоимостью хранения. 
Данные для последнего этапа формировались следующим образом: они распределялись в большую таблицу и становились защищенными, в то время как дополнительно был создан небольшой блок для информации, которая поступала и классифицировалась в течение дня. В этот момент ее можно было обновлять и редактировать, но в итоге данные из нее интегрировались в большую защищенную таблицу, для внесения изменений в которую требовалось огромное количество времени и сил. Поэтому данные в этой таблице стали храниться в статичном неизменном виде (рис. 1).

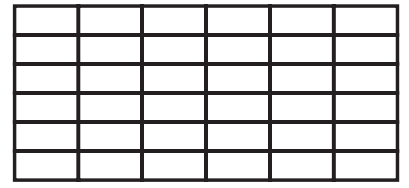

Основная

база данных

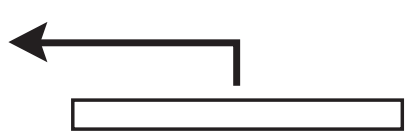

Временная база данных
Рисунок 1. Старый способ хранения информации

Если с локальным производством и системой работы с данными на производстве мы разобрались, необходимо сказать и о том, что происходило в этот период с финансовым сектором в целом. А происходило стремительное развитие финансовых рынков. Инвесторы, малый и средний бизнес стали активно выходить на биржу. Это привело к колоссальным изменениям базовых финансовых показателей компаний. Доля неснижаемого остатка стоимости с 1991 по 2010 г. уменьшилась с более чем $30 \%$ до менее $3 \%$. Широкополосный доступ в Интернет обеспечил участникам биржи возможность работы в реальном времени, возможность работы в боковом тренде. Это привело к финансовой революции - возможности уменьшить спред и повысить ликвидность торгуемых активов, что в свою очередь вызвало увеличение транзакций и рост популярности финансовых рынков. А к 2016 г. на этот рынок вышло население, которое сформировало сегодняшнюю систему взаимодействия на бирже. И к этому времени, конечно, наличие высокопроизводительных сетей, недорогих компьютеров и устройств хранения данных, а также широкое внедрение аппаратной виртуализации, сервис-ориентированной архитектуры и автономных вычислений привели к росту облачных вычислений.

Облачные вычисления - это один из видов интернет-вычислений, который предоставляет общие компьютерные ресурсы и данные обработки для компьютеров и других устройств по запросу. Эта модель обеспечивает повсеместный доступ по требованию к общему пулу конфигурируемых вычислительных ресурсов (например, компьютерных сетей, серверов, хранилищ, приложений и сервисов), которые могут быть быстро подготовлены и выпущены с минимальными затратами управления. Облачные вычисления и решения для хранения данных предоставляют пользователям и предприятиям различные воз- можности для хранения и обработки своих данных либо в частных, либо в сторонних центрах данных, которые могут быть расположены далеко от пользователей. Облачные вычисления позволяют компаниям избежать авансовых расходов на инфраструктуру (например, приобретение серверов). Кроме того, это позволяет организациям сосредоточиться на своем основном бизнесе, а не тратить время и деньги на компьютерную инфраструктуру, также они позволяют предприятиям быстрее и быстрее запускать свои приложения с улучшенной управляемостью и меньшим обслуживанием, а также позволяют командам информационных технологий быстрее адаптировать ресурсы для удовлетворения неустойчивого и непредсказуемого бизнес-спроса.

Широкое распространение в этих условиях получила, например, система SWIFT (Society for Worldwide Interbank Financial Telecommunications - Общество всемирных межбанковских финансовых каналов связи) - сеть обмена сообщениями, которую финансовые учреждения используют для безопасной передачи информации и инструкций через стандартизованную систему кодов. SWIFT присваивает каждой финансовой организации уникальный код, который имеет либо 8 символов, либо 11. Код называется взаимозаменяемым кодом идентификатора банка (BIC), SWIFT-кодом, SWIFT ID или кодом ISO 9362. До SWIFT единственным доступным средством подтверждения сообщений для международных денежных переводов был Telex. Ему мешали низкая скорость, проблемы с безопасностью и формат свободного сообщения - другими словами, y Telex не было единой системы кодов, такой как SWIFT, чтобы назвать банки и описать транзакции. Telex-отправителям приходилось описывать каждую транзакцию в предложениях, которые затем интерпретировались и выполнялись получателем. Это привело к многочисленным ошибкам.

Огромное место в работе организаций стали занимать умные аналитические системы, которые плотно вошли в нашу жизнь [Бабурин, Яненко, 2014]. И хотя они требуют больших затрат при внедрении для сбора статистики (разработки форм и сбора информации), проведения анализа (доработки моделей и разработки отчетов), обслуживания в процессе эксплуатации (резервное копирование и обеспечение защиты), также обследования организации, анализа нормативно-правовой базы, проектирования системы, создания дата-центра, обучения специалистов пользованию и т.д., они все равно остаются на сегодняшний день самыми эффективными. Использование умных систем в сфере финансов позволяет работать с оценкой стоимости и потенциала компании, обеспечивать надежность хранения данных клиентов, проводить точную аналитику сегодняшнего состояния организации, не говоря уже об автоматизации процессов управления и принятии бизнес-решений на операционном уровне [Волкова, 2016]. Появились новые разработки хранения данных. 


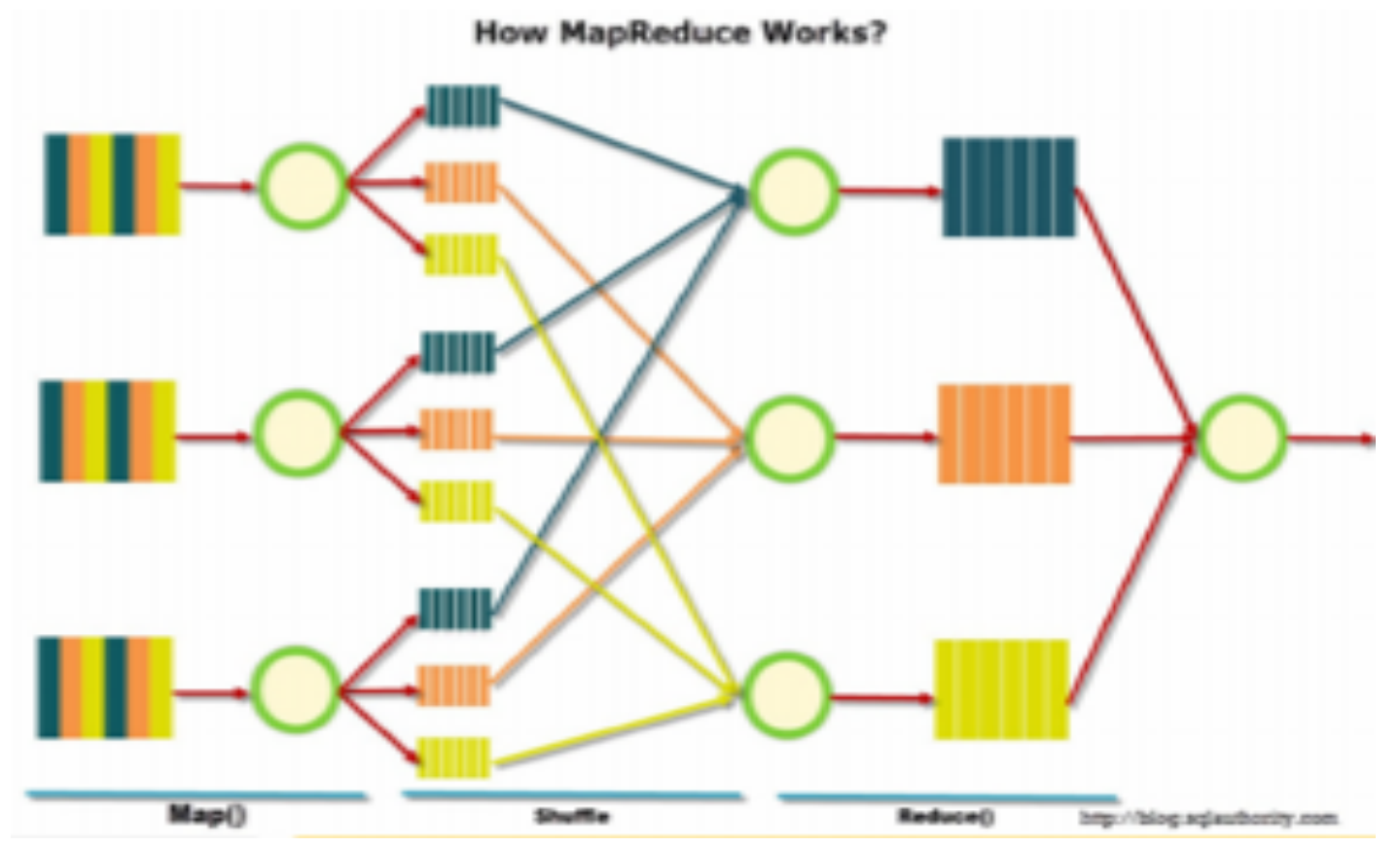

Рисунок 2. Технология Мар Rеdисе. Как структурировать неструктурированные данные

Например, технология Map Reduce, где на первой стадии - Мар - осуществляюся предобработка и фильтрация данных с помощью пользовательской функции, на второй стадии - Shuffle - происходит вывод функции Мар, где данные «разбираются по корзинам», каждая из которых соответствует одному ключу вывода стадии Мар. На завершающем этапе - Reduce - каждая «корзина» со значениями, сформированная на стадии Shuffle, попадает на вход функции Reduce.

Появилась также технология блокчейн, которая представляет собой определенно структурированную базу данных и имеет определенные правила осуществления цепочек транзакций. Пока еще эта система очень хорошо способна обеспечивать безопасность вложенных данных и предотвращать случаи мошенничества. Эта система открыта в одностороннем порядке, т.е. обеспечить ее данными может любой пользователь, наличие определенных данных также может увидеть каждый желающий, но осуществление операций возможно только с разрешения майнеров, которые наделены определенными правами подтверждения осуществления транзакции. Информация попадает в систему с одним кодом, трансформируется и сохраняется с другим, распределяется с третьим. Для разрешения майнеров тоже необходим определенный ключ, подтверждающий или запрещающий операцию. Первоначально на базе блокчейна сформировалась платежная система «Биткоин» для возможности функционирования криптовалюты. Ведь если сравнить систему блокчейн с ДНК, то каждая клетка - каждый блок информации - является отражением всей системы и полностью завязана с другими элементами. Это значит, что в систему нельзя ни доба- вить ничего лишнего, ни незаметно исправить. Если раньше система использовалась исключительно для транзакций с криптовалютой, то сегодня начинает развиваться в банковском секторе.

Появляются платформы для разработки бизнес-приложений на основе технологии блокчейн, которые могут контролировать даже соблюдение авторских прав, отслеживать процессы производства того или иного товара на его соответствие заявленным стандартам, не говоря уже абсолютно обо всех финансовых операциях.

Все указанные процессы, системы, технологии являются результатом не только увеличения объемов данных, но и возникновения принципиально новых форматов. В этих условиях сложились основные принципы работы с Big Data: принцип горизонтальной масштабируемости, отказоустойчивости и локальности данных. Главное, что эти принципы позволяют решать такие задачи современных технологий Big Data, как классификация, кластеризация (распределение по принципу «похожести»), сокращение описания (для возможностей быстрого и емкого информирования, визуализации), ассоциация, прогнозирование, анализ отклонений. Среди методов, позволяющих решать эти задачи, можно назвать и кластерный анализ, анализ выбросов, анализ скрытых закономерностей и, конечно, эволюционные алгоритмы, которые запускают весь этот процесс. Несмотря на то что основные возможности хранения данных кардинально не изменились, появились только новые механизмы, классификаторы, нейросети, их появление связано прежде всего с принципиальным изменением структуры и типов данных. Больше не существует ограничений стандартными графиче- 
скими, текстовыми и числовыми ресурсами. Сегодня приходится иметь дело с новыми данными. К ним относятся фото-, видео-, аудиоформаты, также специфические данные, психологические и поведенческие характеристики клиентов, их субъективная оценка, закодированная тактильная информация, которую необходимо не просто распознавать, но анализировать и принимать на основе анализа бизнес-решения.

Невозможно отрицать, что наша жизнь развивается в период экономики знаний. Лидирующие страны мира, такие как США, Япония и Германия, начали этот переход еще в 1980-х гг. Однако Россия в связи с событиями 1990-х гг. - кризисом и дефолтом - начала несколько отставать от них, поэтому наблюдается подобная трансформация экономики сегодня, причем переход происходит колоссальными темпами. В связи с этим возникает вопрос: готова ли Россия к развитию экономики знаний?

Характеристики постиндустриальной экономики:

1. производительность все в большей степени зависит от использования достижений науки и техники, а также от качества информации и менеджмента;

2. в развитых капиталистических странах внимание производителей и потребителей смещается от материального производства в сторону информационной деятельности;

3. происходит глубокая трансформация организации производственного процесса (от стандартизированного массового в сторону «кастомизированного» производства и от вертикально интегрированной организации в сторону горизонтальных сетевых взаимоотношений и взаимосвязей между подразделениями);

4. экономика становится все более глобальной, вследствие чего капитал, производство, менеджмент, рынки, труд, информация и технологии организованы вне зависимости от национальных границ;

5. технологические изменения, в основе которых информационные технологии, преобразующие материальную основу современного мира, приобретают все более революционный характер.

Следствием перехода к экономике знаний является прежде всего возросшая ценность информации, которая становится главным товаром. Удивительное свойство ограниченности ресурсов не распространяется на информацию, она становится все более доступной. Тем не менее проблема поиска информации является ключевой в современной реальности. Google и прочие общедоступные поисковые системы при стандартном подходе к поиску не дают возможности найти необходимую информацию. Поисковый робот выдает только 1-2\% всей собранной информации, при этом лишь порядка 10\% являются релевантными запросу. Введя один и тот же запрос в одно и то же время, мы получаем не одинаковый результат. Google анализирует данные о владельце персонального компьютера (ПК) таким образом, что предоставляет ему ту информацию, которую пользователь хочет увидеть, но не ту, которая ему нужна.

Для решения подобных регулярно возникающих проблем, во-первых, можно использовать поисковые операторы, которые позволят построить более точный запрос.

Во-вторых, можно воспользоваться специальными поисковыми программами, которые дают более релевантные результаты. iMetaSearch - поисковая программа, позволяющая получить поисковые результаты, максимально адекватные запросу, сгруппированные по категориям и ключевым словам. В бесплатной версии работает Google, в платной версии может быть подключено до 16 «поисковиков». «Нейрон» включает надстройку для объединения поиска Google и Yandex, а также позволяет осуществлять конкурентный анализ.

И третий, самый лучший, но и самый дорогостоящий вариант - создание собственного поискового робота, который способен удовлетворить специализированные запросы наиболее полно и точно.

Помимо этого, существуют методы автоматизации и упрощения информации:

\section{- мониторинг финансовых веб-ресурсов:}

- «Аваланче 2.5» - мониторинг выбранных интернет-ресурсов, группировка результатов поиска по различным параметрам;

- WebSite-Watcher - мониторинг веб-страниц, включая защищенные паролем, мониторинг форумов, RSS-каналов, групп новостей, локальных файлов (ведется автоматически, поставляется в удобном для пользователя виде);

- Check\&Get - интернет-органайзер, система мониторинга веб-сайтов и офлайновый браузер;

\section{- анализ информации:}

- $\quad$ http://www.atlasti.com - единая информационная среда для работы с различными текстовыми, табличными, аудио- и видеофайлами как единым целым, а также инструменты качественного анализа и визуализации;

- $\quad$ http://netcomber.com - сервис по анализу веб-сайтов и установлению их связей с родственными ресурсами;

- $\quad$ http://www.la0.ru - удобный и релевантный сервис анализа ссылок и бэклинков на интернет-ресурс;

- Sentinel Vizualizer - визуализация связей и отношений;

- $\quad$ http://www.analytictech.com/ucinet - анализ и визуализации социальных сетей (хорошо использовать для анализа взаимосвязи компаний и лиц, выявления неформальных групп); 


\section{- сбор информации:}

- $\quad$ http://www.bigvisor.ru - сервис отслеживания рекламных кампаний конкурентов;

- $\quad$ http://www.rivaliq.com - инструмент для ведения конкурентной разведки на западных, в первую очередь европейских и американских, рынках товаров и услуг;

- $\quad$ http://www.advse.ru - позволяет анализировать рекламные кампании конкурентов;

- $\quad$ http://www.recipdonor.com - сервис позволяет осуществлять автоматический мониторинг всех действий на сайтах конкурентов;

- $\quad$ http://www.shodanhq.com - определяет IP-адреса, типы роутеров, компьютеров, серверов и рабочих станций, размещенных по тому или иному адресу, прослеживает цепочки DNS-серверов и позволяет реализовать много других интересных аналитических функций.

Студенты должны обладать знаниями о возможностях современных технологий Big Data и уметь их применять.

Что касается задач фундаментальных наук, то на проблему поиска обращают внимание передовые мировые школы бизнеса, такие как NYU Stern, Wharton, Chicago Booth и Stanford. Они понимают, что необходимо научить молодых специалистов правильно пользоваться технологиями Big Data, поэтому вводят изменения в образовательный процесс. Современный экономист не обязан глубоко погружаться в тонкости технологий, позволяющих обрабатывать и анализировать большие данные, но должен понимать основы, так как его целью, как и задачей менеджера, является правильная постановка задачи, а построением алгоритмов и созданием программ занимаются профильные специалисты: математики и программисты.

Сегодняшним действенным и популярным методом классификации и прогнозирования является метод деревьев решений (decision trees). Это способ представления правил в иерархической последовательной структуре. Основа такой структуры - ответы «да» или «нет» на ряд вопросов. Алгоритмы конструирования деревьев решений состоят из этапов создания дерева (tree building) и сокращения дерева (tree pruning). В ходе создания дерева решаются вопросы выбора критерия расщепления и остановки обучения (если это предусмотрено алгоритмом). В ходе этапа сокращения дерева решается вопрос отсечения некоторых его ветвей. Так же широко применяется метод Dynamic Quantum Clustering (DQC). Он работает без предварительного знания о тех структурах, их типе и топологии, которые могут быть скрыты в данных и выявлены в результате его применения. Парадигма - пусть данные говорят о себе сами. Задача - «как искать иголку в многомерном стоге сена, не зная, как она выглядит, и не зная, есть ли она в этом стоге». Метод хорошо работает с многомерными данными. Время анализа линейно зависит от размерности.
Знание указанных методов анализа, а также знания о возможностях совершенствования модернизации этих методов позволяют расширять использование Big Data. Поэтому передовые университеты считают обязательным для экономистов владеть языками программирования SQL (он применяется для создания баз данных) и R (позволяет вести анализ данных).

Прикладные дисциплины также необходимы для современных экономистов. Условия экономики знаний, процессов глобализации и интеграции культур не только расширили возможности взаимодействия государств, передачу опыта и т.д., но и многократно усилили конкуренцию и изменили поведение как потребителей, так и производителей. Все это не только справедливо, но и очень значимо для финансового сектора.

Несомненно, сегодня наиболее ценным является обладание уникальной информацией, что имеет следующее значение с эмпирической точки зрения: грамотное управление и применение знаний. Фокус внимания современных компаний направлен на клиента. Мы отошли от устаревшей маркетинговой стратегии 4P (Product, Place, Price, Promotion) и перешли к маркетинговой стратегии 4С (Consistency, Content, Convenience, Contextual), более того, это стало единой концепцией C-Customer.

Главной и основной ценностью сегодняшних компаний стал клиент. В тренде клиент-ориентированность организаций. В таких успешных клиентоцентричных компаниях все большую роль начали играть нематериальные активы, подтверждающие ценность обладания знаниями и уникальной информацией. С точки зрения анализа данных о клиенте, данных, полученных в процессе выстраивания доверительных долгосрочных отношений, это маркетинговые активы.

Если раньше, в период pull-экономики, фирмы просто производили товар и представляли его на рынке, учитывая общие тенденции запросов общества, то в push-экономике, где клиент имеет огромный выбор, компании, чтобы удержать потребителей, должны искать к ним индивидуальный подход и формировать уникальное торговое предложение с учетом предпочтений клиента. Формирование представления о жизни клиента в pull-экономике необходимо для создания единой с потребителем экосреды. Степень доверия и лояльности клиента определяет его выбор. Цена, упаковка, даже функционал отходят на второй план по сравнению с крепкими налаженными отношениями. Формирование привычки, создание удобных условий, уважительное обращение, предвосхищение проблем и потребностей создают эффективное поле для удержания клиента, которое формирует у клиента не просто приверженность, но привычку, создает зону комфорта. Кроме того, возросла роль партнерств. Если говорить, например, о компании Samsung, то производство фирмы практически полностью основано на аутсорсинге. Производство деталей, сборка, логистика и т.д. осуществляются за 
физическими пределами компании, значит, серьезно упрочилось сотрудничество, расширились сети контактов и связей. Так сформировались три базовых элемента нематериальных активов: маркетинговые активы, партнерство и бизнес-интеллект. Конечно, все это способно эффективно функционировать при условии наличия сильного конкурентного бренда. Роль управления всеми указанными процессами и возможностями возрастает настолько, что именно знания становятся основным конкурентным преимуществом компании на современном рынке.

Важно сказать, что, несмотря на такое значительное изменение конъюнктуры, цель компаний по-прежнему состоит в увеличении собственной стоимости, повышении своей инвестиционной привлекательности, развитии и расширении своего бизнеса. Тренды исследований таких мировых лидеров экономики финансов, как Kellogg, Stanford, HBS, основаны на трех основных темах: знания, управление и инновации. Сюда можно отнести генерацию знаний (наука и ее сегменты в других секторах) - Гарвард, Университет Чикаго, Принстон; распространение и применение знаний (исследования и разработки - ИиР, производство товаров и услуг, коммерциализация нововведений) - Массачусетский технологический институт, Гарвард; рынок научно-технической продукции, рыночные институты - Стэнфорд; области исследований экономического факультета Университета Чикаго сегодня (прикладная микроэкономика, эконометрика, экономическая теория, макроэкономика); образование и профессиональная подготовка кадров - Кэллог, программа MBA, MBA для руководителей, Калифорнийский университет; инновационная инфраструктура, включая финансовое обеспечение, - Кэллог, программа МММ, бизнес и инновации, технологии, программы Гарварда о технологиях, инновациях и образовании; управление и регулирование (правовая база, государственная макроэкономическая и инновационная политика, корпоративное управление, рыночные механизмы) Кэллог, двойные программы JD-MBA.

Развитие этих трендов и появление двойных программ связано с расширением потоков информации, которыми мы можем оперировать, при этом формируя лучшее качество. Правильная приоритизация, кластеризация, распределение информации позволяют получать реальный экономический эффект.

Перечисленные научные и бизнес-школы видят ценность в воспитании осведомленных во всех отраслях специалистов узкого профиля. Это необходимо для формирования навыка постановки правильных запросов и понимания ключевых задач. Научные лидеры в области менеджмента и маркетинга видят сегодняшнюю основную проблему в неточности построения прогнозов развития бизнеса, которые помогают принимать современные решения на основе оценки и прогнозов будущей стоимости и инвестиционной привлекательности компаний. Основное решение предлагается в новом подходе к обработке данных, формализации процессов постановки задачи. Стандартные методы и алгоритмы работы на этапах сбора, хранения, интерпретации, анализа данных устарели, поскольку они работают с данными старого типа.

Для работы с новыми данными сегодня предлагаются когнитивные технологии. Они помогают значительно увеличить скорость обработки данных. Помимо этого, в отличие от привычных «поисковиков», которые выдают очень ограниченный перечень источников информации в ответ на наш запрос, когнитивные технологии способны на основе анализа непрерывно обновляющихся баз данных сформулировать решение и предоставить его в ответ на запрос [Блинов, Рахманкулов, 2015].

Разработками таких когнитивных технологий, внедряемых в производство, занимаются крупнейшие клиент-ориентированные корпорации, такие как IBM, Microsoft, Google и др. Можно перечислить многие системы: Microsoft Data, Yandex Data Factory, SAP HANA, Google Now и Siri, PROMT Analyser. Например, компания Citygroup работает с продуктом IBM Watson, который применяется в финансовом менеджменте для помощи финансистам в принятии бизнес-решений [Brighten your day with cognitive UEM, 2017].

Таким образом, обучение современных финансистов должно ориентироваться на исследования западных бизнес-школ, на работу крупнейших и успешных компаний, быть основано на работе с актуальной информацией, с усовершенствованными аналитическими методами, применяемыми в маркетинговой деятельности клиент-ориентированных организаций, использовании при этом элементов стратегического, статистического, математического, системного, стохастического, вероятностного и других видов анализа. Среди них: А/B-тестирование, паттерн-анализ, изучение правил ассоциации, предиктивное моделирование, принципы классификации, построение и анализ регрессии, кластерный анализ, анализ настроений, методы объединения и интеграции данных, методы обработки сигналов, ансамблевое обучение, пространственный анализ, изучение генетических алгоритмов, статистика, машинное обучение, контролируемое и самостоятельное обучение, языки программирования, симуляция, изучение процессов нейронных сетей, анализ временных рядов, анализ взаимосвязей, способы визуализации.

Важно, что совокупной работой прикладных и фундаментальных наук должны быть обучение студентов эффективной работе с данными, формирование более глубокого представления финансовых процессов и процессов других отраслей, которые интегрируют и в финансовый сектор. Конкурентным преимуществом грамотного финансового специалиста должна являться невозможность субсидирования его знаний и навыков их применения. Новые идеи, новые запросы с использованием новых технологий обязывают опережать время результативными экономическими решениями. 


\section{Список литературы}

1. Бабурин B.A., Яненко M.E. (2014) Технологии Big Data в сервисе: новые рынки, возможности и проблемы // Технико-технологические проблемы сервиса (ТТПС). № 1 (27). С. $100-105$.

2. Блинов А.О., Рахманкулов И.Ш. (2015) Управление организациями с помощью когнитивных технологий // Вестник Уфимского государственного нефтяного технического университета (УГНТУ). Наука, образование, экономика. Серия: Экономика. № 4 (14). С. 33-39.

3. Быкова А.А, Морковкина Е.В. (2013) Отношенческий капитал как фактор повышения стоимости компании // Корпоративные финансы. № 4 (28). С. 19-36.

4. Волкова Ю.С. (2016) Большие Данные в современном мире // Концепт. Т. 11. C. $1171-1175$.

5. 5. Кузубов С.А. (2014) Развитие концептуальных подходов к измерению гудвилл с исторической перспективы // Корпоративные финансы. № 3 (31). С. 94-104.

6. Макеева Е.Ю., Аршавский И.В. (2014) Применение нейронных сетей и семантического анализа для прогнозирования банкротства // Корпоративные финансы. № 4 (32). С. 130-141.

7. Мальцева С.В., Лазарев В.В. (2015) Маркетинговая аналитика в сфере электронного бизнеса на основе больших данных // Информационные технологии в проектировании и производстве. № 1. С. 62-67.

8. Поколение ЭВМ (2007). Режим доступа: http://pchistory.narod.ru/pokoleniya.html (дата обращения: 10.02.2017).

9. Al Powell, Harvard Staff Writer (2017). Bringing big data to the farm. Available at: http://news.harvard.edu/gazette/story/2017/04/the-future-of-food-will-be-proactive-efficientand-digitized-or-else/ (accessed: 09.02.2017).

10. Alyson Y. (2017) Our partnership with Imperial College London: The Digital Asset Research Lab. Available at: https://blog.blockchain.com/2017/01/19/our-pare ем tnership-withimperial-college-london-the-digital-asset-research-lab/ (accessed: 11.02.2017).

11. Brighten your day with cognitive UEM (2017) Available at: http://www-03.ibm.com/security/ mobile/maas360.html (accessed: 09.02.2017).

12. Polonski V. (2014) The evolution of social networking sites: The rise of content-centric platforms which favour the perpetual present. Available at: http://www.kellogg.ox.ac.uk/blog/the-evolution-of-social-networking/ (accessed: 11.02.2017).

13. United Nations (2016) World Economic Situation and Prospects. Available at: http://www.unic.ru/sites/default/files/Доклад\%20WESP2016.pdf (accessed: 11.02.2017). 


\section{References}

1. Baburin V.A., Yanenko M.E. (2014) Tekhnologii Big Data v servise: novye rynki, vozmozhnosti i problemy [Technologies of Big Data in a service: New markets, capabilities, and promlems]. TTPS, no. 1 (27), pp. 100-105. (In Russ.)

2. Blinov A.O., Rakhmankulov I.Sh. (2015) Upravlenie organizatsiyami s pomoshch'yu kognitivnykh tekhnologii [The management of organizations with the help of using cognitive technologies]. VESTNIK UGNTU. Science, education, economy. Series: The Economy, no. 4 (14), pp. 33-39. (In Russ.)

3. Bykova A.A., Morkovkina E.V. (2013) Otnoshencheskyi kapital kak factor povysheniya stoimosti kompanii [The capital of relationships as a factor of rising value of an organization]. Journal of Corporate Finance Research, no. 4 (28), pp. 19-36. (In Russ.)

4. Volkova U.S. (2016) Bol'shie dannye v sovremennom mire [Big data in the modern world]. Concept, vol. 11, pp. 1171-1175. (In Russ.)

5. Kuzubov S.A. (2014) Razvitiye kontseptual'nikh podkhodov k izmereniyu goodwill s istoricheskoi perspektivy [The development of conceptual approaches to changes of goodwill from the historical]. Journal of Corporate Finance Research, no. 3 (31), pp. 94-104. (In Russ.)

6. Makeeva E.Yu., Arshavskiy I.V. (2014) Primenenie neironnykh setei i semanticheskogo analiza dlya prognozirovaniya bankrotstva [An application of neural networks and semantic analysis for forecasting bankruptcy]. Journal of Corporate Finance Research, no. 4 (32), pp. 130-141. (In Russ.)

7. Maltseva S.V., Lazareva V.V. (2015) Marketingovaya analitika v sphere electronnogo biznesa na osnove bol'shikh dannykh [The marketing analytics in the area of electro business on a base of Big Data]. Information Technologies in Modeling and Manufacturing, no. 1, pp. 62-67. (In Russ.)

8. Pokoleniye EVM [Computer's generation] (2007) Available at: http://pchistory.narod.ru/pokoleniya.html (accessed: 10.02.2017). (In Russ.)

9. Al Powell, Harvard Staff Writer (2017) Bringing big data to the farm. Available at: http://news.harvard.edu/gazette/story/2017/04/the-future-of-food-will-beproactive-efficient-and-digitized-or-else/ (accessed: 10.02.2017).

10. Alyson Y. (2017) Our partnership with Imperial College London. The Digital Asset Research Lab. Available at: https://blog.blockchain.com/2017/01/19/our-partnership-with-imperialcollege-london-the-digital-asset-research-lab/ (accessed: 11.02.2017).

11. Brighten your day with cognitive UEM (2017) Available at: http://www-03.ibm.com/security/mobile/maas360.html (accessed: 09.02.2017).

12. Polonski V. (2014) The evolution of social networking sites: The rise of content-centric platforms which favour the perpetual present. Available at: http://www.kellogg.ox.ac.uk/blog/the-evolution-of-social-networking/ (accessed: 11.02.2017).

13. United Nations (2016) World Economic Situation and Prospects. Available at: http://www.unic.ru/sites/default/files/Доклад\%20WESP2016.pdf (accessed: 09.02.2017). 